\title{
Consultas odontológicas de mujeres consumidoras de cannabis y/o cocaína versus no consumidoras. Estudio de cohorte retrospectivo durante 4 años en Argentina
}

\author{
Dental attendance of women cannabis and/or cocaine users versus \\ non users. Retrospective cohort study during 4 years in Argentina \\ Consultas odontológicas em mulheres usuárias de cannabis e/ou cocaína contra \\ não usuárias. Estudo de coorte retrospectivo durante 4 años em argentina
}

\author{
Marina Villarreal' ${ }^{1 D}$ 0000-0003-4934-8481 \\ Valeria Belmonte ${ }^{2}$ (D) 0000-0003-2139-0645 \\ Jorge Luis Olivares ${ }^{1}$ (D) 0000-0002-2010-4048 \\ Claudia López 1 (D) 0000-0002-3075-6306 \\ María Paula Aguilera ${ }^{3}$ (iD) 0000-0002-7322-1221
}

\section{Resumen}

Introducción: El consumo de cannabis y de cocaína constituye un problema global que afecta la salud bucal. La mayoría de las investigaciones se han realizado en hombres, en programas de rehabilitación.

Objetivo: describir y comparar consultas y diagnósticos odontológicos de mujeres consumidoras y no consumidoras de cocaína y/o cannabis, por un período de 4 ańos.

Métodos: estudio de cohorte retrospectivo. Grupos comparados de mujeres consumidoras y no consumidoras de cannabis y/o cocaína, seleccionadas en el posparto en un hospital público.

Resultados: la edad promedio en ambos grupos fue de 22 años, sin diferencias en nivel educativo. Las mujeres del grupo de consumidoras $(n=29)$ fueron mayormente policonsumidoras, realizaron más consultas odontológicas de emergencias $(\mathrm{p}=0,0002)$, y presentaron más gingivitis y periodontitis $(\mathrm{p}=0,0001)$ que las no consumidoras $(\mathrm{n}=58)$.

Conclusiones: las mujeres consumidoras de cannabis y/o cocaína realizaron más consultas por emergencias, y presentaron con mayor frecuencia diagnóstico de gingivitis y periodontitis que las no consumidoras.

Palabras clave: salud bucal, cannabis, cocaína, consultas, enfermedad periodontal.

1 Departamento de Docencia e Investigación. Establecimiento Asistencial Dr. Lucio Molas, La Pampa, Argentina. marvillarreal@ cpenet.com.ar

2 Cátedra de estadística. Facultad de Agronomía. Universidad Nacional de La Pampa, La Pampa, Argentina

3 Servicio de Laboratorio químico. Establecimiento Asistencial Dr. Lucio Molas, La Pampa, Argentina 


\section{Abstract}

Introduction: The use of cannabis and cocaine is a global problem that affects oral health. Most of the research has been performed on men, in rehabilitation programs.

Objective: to describe and compare dental attendances and oral diagnoses of women cannabis and/or cocaine users and non users, during 4 years.

Methods: retrospective cohort study. Compared groups of women who use and do not use cannabis and/or cocaine, selected in the postpartum period in a public hospital.

Results: the average age in both groups was 22 years, without educational level differences. Women in the consumers group $(n=29)$ were mostly polyconsumers, made more urgency dental attendances $(\mathrm{p}=$ 0.0002 ), and presented more gingivitis and periodontitis $(\mathrm{p}=0.0001)$ than non-users $(n=58)$.

Conclusions: women who used cannabis and/or cocaine made more attendances for oral urgencies, and had a more frequent diagnosis of gingivitis and periodontitis than non-users.

Keywords: oral health, cannabis, cocaine, attendance, periodontal disease.

\section{Introducción}

El consumo de cannabis y de cocaína constituye un problema global, ya que provoca diversas consecuencias adversas para la salud individual, familiar y comunitaria. ${ }^{(1)}$ Luego del alcohol y del tabaco, cannabis y cocaína se encuentran entre las drogas psicoactivas (DP) consumidas con más frecuencia por adolescentes y adultos en la mayoría de los países del mundo. ${ }^{(1,2,3)}$

Argentina no es la excepción. Un estudio en 2017, mostró que, luego de tabaco y alcohol,

\section{Resumo}

Introdução: $\mathrm{O}$ uso de cannabis e cocaína é um problema global que afeta a saúde bucal. A maior parte da pesquisa foi feita em homens, em programas de reabilitação.

Objetivo: descrever e comparar consultas e diagnósticos odontológicos de mulheres que consumiram e não consumiram cocaína e/ ou maconha, durante 4 anos.

Métodos: estudo de coorte retrospectivo. Grupos comparados de mulheres que usam e não usam cannabis e/ou cocaína, selecionados no período pós-parto em um hospital público.

Resultados: a média de idade em ambos os grupos foi de 22 anos, sem diferenças de escolaridade. As mulheres do grupo das consumidoras $(n=29)$ eram, em sua maioria, policonsumidoras, realizavam mais consultas odontológicas de urgência $(\mathrm{p}=0,0002)$ e apresentavam mais gengivite e periodontite $(\mathrm{p}=0,0001)$ do que as não usuárias $(\mathrm{n}=58)$.

Conclusóes: mulheres usuárias de cannabis e/ou cocaína realizaram mais consultas de urgência e tiveram diagnóstico de gengivite e periodontite mais frequentes do que as não usuárias.

Palavras-chave: saúde bucal, cannabis, cocaína, consultas, doença periodontal.

cannabis y cocaína son las DP de mayor consumo en la población general, con aumentos exponenciales en la última década en ambos sexos. Los mayores porcentajes de consumo y de incremento se encuentran en jóvenes entre 18 y 24 años. ${ }^{(3)}$

En términos generales, las consecuencias sobre la salud bucal de los consumidores de DP son muy variadas. Pueden afectar los tejidos blandos y duros de la boca, inducir malignidad y predisponer a infecciones. ${ }^{(4)}$ Las alteraciones descriptas con mayor frecuencia son la enfer- 
medad periodontal, caries y pérdidas dentarias, que suelen relacionarse con mayor tiempo de consumo. ${ }^{(4,5,6)}$ La coexistencia de una dieta altamente cariogénica, y/o de complejos factores de riesgo, individuales, familiares y sociales, inciden en la génesis de la mala salud bucal. (2) Dichos factores, que incluyen a la frecuente modalidad de policonsumo de DP, dificultan el diagnóstico, el abordaje terapéutico y la investigación en los pacientes afectados. ${ }^{(4,7)}$

Respecto de cannabis, se ha demostrado la relación entre su consumo y el desarrollo de enfermedad periodontal. ${ }^{(8-11)} \mathrm{La}$ asociación entre fumar cannabis y otras alteraciones como caries, lesiones de partes blandas y cáncer oral, no ha sido consistente. ${ }^{(9)}$ Se ha descripto que el estado mental de los fumadores de cannabis puede demorar las consultas para tratamiento. ${ }^{(9)}$

En relación a cocaína, la frecuencia de periodontitis, placa visible, sangrado gingival y lesiones de la mucosa oral fue significativamente mayor en consumidores que en no consumidores. ${ }^{(5,12)}$ En otros estudios, la presencia de sarro y una mayor profundidad de sondeo fueron los hallazgos encontrados más frecuentemente. ${ }^{(6,13)}$ La amplia mayoría de estudios sobre salud bucal en consumidores de DP se refieren a hombres, que en general se encuentran en unidades de tratamiento o en programas de rehabilitación, y/o carecen de grupo de comparación. ${ }^{(4,6,12-14)}$

El objetivo de este estudio es describir y analizar los tipos de consulta odontológica y los diagnósticos odontológicos de mujeres consumidoras de cannabis y/o cocaína durante un período de 4 años, y comparar con mujeres no consumidoras.

\section{Métodos}

Estudio de cohorte retrospectivo. Las mujeres fueron seleccionadas del sector de internación conjunta del Servicio de Neonatología del Establecimiento Asistencial Dr. Lucio Molas (EALM) de Santa Rosa La Pampa Argentina, en el posparto inmediato.
Mujer consumidora de cocaína y/o cannabis (MC): se incluyeron todas las mujeres con criterio de detección y muestra en orina posparto positiva para cocaína y/o cannabis, realizada en el Servicio de Neonatología del EALM, entre 2009 y 2013.

Mujer no consumidora de cocaína ni de cannabis (MNC): mujer sin criterio de detección, con parto en el mismo Servicio de Neonatología en mismo día o semana que una MC. Este grupo de comparación fue seleccionado considerando similar edad materna, localidad de procedencia, y situación ante la seguridad social que el grupo MC. Se seleccionaron dos mujeres no consumidoras por cada mujer consumidora, para aumentar la potencia del estudio.

\section{Criterios y método de detección de DP en orina}

Se solicitó ante la presencia de criterios prefijados para el posparto inmediato, (comunicación actual o historia de uso de drogas, estado mental alterado, ausencia de control del embarazo, complicaciones del sistema nervioso central inexplicables o síntomas compatibles con abstinencia en el recién nacido). Cada mujer firmó un consentimiento, y obtuvo su orina en vaso recolector. La orina se analizó con prueba rápida en tira reactiva para la detección cualitativa simultánea de drogas o metabolitos: anfetaminas, barbitúricos, benzodiacepinas, tetrahidrocannabinol, cocaína, metadona, metanfetaminas, opiáceos y fenciclidina. La prueba de multidrogas en un solo paso en Panel ABON realizada, es un inmunoensayo cromatográfico de flujo lateral.

\section{Proceso de incorporación y de seguimiento de las cohortes}

Cada mujer con resultado positivo en orina para cocaína y/o cannabis y sus 2 controles se incorporaron en una base de datos de investigación durante los años 2009 a 2013. No se realizó evaluación odontológica en el posparto. El seguimiento de las consultas odontológicas 
realizadas por las mujeres durante 4 años fue retrospectivo, revisando los registros de historias clínicas digitales del Sistema Informático de Salud pública de la provincia de La Pampa (SIS). Las consultas de mujeres incorporadas al estudio en 2009 se revisaron hasta 2013, las de 2010 hasta 2014, y del mismo modo se realizó para las incorporadas en los siguientes años.

\section{Variables analizadas}

Nivel educativo; enfermedades previas y adquiridas; tipo de drogas detectadas en orina; edad de inicio del consumo; tiempo de consumo; consumo de tabaco y alcohol; tipo de consultas odontológicas (control= con turno programado, o urgencia $=$ sin turno previo); número de consultas por mujer; diagnósticos odontológicos según CIE-10; internaciones por patología bucal.

\section{Fuentes}

Registro propio de investigación, y Sistema Informático de Salud (SIS) de La Pampa: en esta base de datos se registran datos demográficos e historia clínica de los individuos asistidos en todos los establecimientos públicos de salud de la Provincia, en las distintas especialidades médicas y en odontología. Los diagnósticos están codificados según la Clasificación Internacional de Enfermedades (CIE-10).

No hubo faltantes de datos en las variables que se presentan.

\section{Procesamiento de datos y estadística}

Para la carga, procesamiento inicial y gráficos se utilizó Excel de Office 4.0. Para el análisis estadístico se utilizó InfoStat 2019, Universidad de Córdoba. ${ }^{(15)}$

Se realizó estadística descriptiva: frecuencias absolutas y relativas para las variables cualitativas y medidas de tendencia central y de dispersión para las variables cuantitativas. Para la fase comparativa se utilizaron las pruebas de chi-cuadrado (variables cualitativas) o pruebas de comparación de medias, t-Student (variables cuantitativas). Se consideró diferencia estadísticamente significativa un valor de $p<0,05$. Las tasas se expresan como riesgo relativo (RR) y su intervalo de confianza del 95\% (IC95\%).

Consideraciones éticas: estudio aprobado por el Comité de Ética en Investigación del EALM (número de registro: 03/2016). La confidencialidad se mantuvo codificando y limitando la búsqueda, registro, análisis y acceso a la base de datos del estudio a los investigadores.

\section{Resultados}

No hubo diferencias significativas entre mujeres consumidoras (MC) y mujeres no consumidoras (MNC) en las variables de selección, siendo los grupos homogéneos (Tabla 1). La edad promedio tanto de las mujeres consumidoras (MC) como de las no consumidoras (MNC) fue de 22 ańos (DE MC:4,78; MNC:4,33).

Tabla 1: Comparación de MC y MNC según variables de selección. Mujeres asistidas posparto en el Servicio de Neonatología del Establecimiento Asistencial Dr. Lucio Molas, La Pampa, entre 2009 y 2013

\begin{tabular}{|c|c|c|c|c|}
\hline & \multicolumn{2}{|c|}{ Grupos de mujeres } & Comparación entre grupos \\
\hline & & MC & MNC \\
\hline & & $(n=29)$ & $(n=58)$ & $p=0,64$ \\
\hline Edad materna (años) & Media (DE) & $22(5)$ & $22(4)$ & $p=0,165$ \\
\hline Residencia Santa Rosa /Toay & (IC 95\%) & $21-24$ & $23-39$ & \\
\hline Obra social /prepaga & & 29 & 53 & no \\
\hline
\end{tabular}

MC: mujeres consumidoras de cocaína y/o cannabis en embarazo. MNC: mujeres no consumidoras de esas drogas en el embarazo.

DE: desvío estándar; IC 95\%: Intervalo de confianza del 95\% 
No se encontraron diferencias significativas en el nivel educativo entre los grupos $(\mathrm{p}=0,32)$, siendo el nivel secundario incompleto el predominante (Tabla 2).

Tabla 2: Nivel educativo de MC y MNC. Mujeres asistidas posparto en el Servicio de Neonatología del Establecimiento Asistencial Dr. Lucio Molas, La Pampa, entre 2009 y 2013

\begin{tabular}{|c|c|c|}
\hline Nivel educativo & MC $(n=29)$ & MNC $(n=58)$ \\
\hline Primario Incompleto & 0 & $1(2 \%)$ \\
\hline Primario Completo & $10(34 \%)$ & $13(22 \%)$ \\
\hline Secundario Incompleto & $17(59 \%)$ & $33(57 \%)$ \\
\hline Secundario Completo & $2(7 \%)$ & $11(19 \%)$ \\
\hline
\end{tabular}

MC: mujeres consumidoras de cocaína y/o cannabis en embarazo.

MNC: mujeres no consumidoras de esas drogas en el embarazo

Ninguna mujer en ningún grupo tuvo diabetes, HIV, ni enfermedad inmunosupresora previa al embarazo, adquirida durante el mismo ni durante 4 años posparto.

Las drogas detectadas en orina de MC fueron: cocaína $9(31 \%)$, cannabis $10(35 \%)$, cocaína y cannabis 5 (17\%), cocaína con fenciclidina y/o benzodiazepinas 5 (17\%).

El rango de edad en que iniciaron el consumo de cocaína y/o cannabis fue de 11 a 29 años. El tiempo promedio de consumo fue de 5,14 años (DE:2,96). El 93\% fueron policonsumidoras durante el embarazo, asociando principalmente cocaína y/o cannabis con tabaco y/o alcohol. Relataron haber consumido tabaco durante el embarazo 93\% de estas mujeres, y 21\% de las MNC ( $p<0,0001)$. El 55\% de las MC había tomado alcohol durante el embarazo, en tanto que ninguna MNC lo había hecho ( $\mathrm{p}<0,0001)$. El tipo y frecuencia de consultas odontológicas realizadas por ambos grupos de mujeres durante 4 años se muestran en la Tabla 3. Puede observarse que no hubo diferencias entre grupos en el porcentaje de mujeres que realizaron consultas de control, pero hubo significativa diferencia en las consultas por urgencias odontológicas, en que las MC superaron a las MNC.

Tabla 3: Consultas odontológicas durante 4 años. Comparación entre MC y MNC, ingresadas al estudio entre 2009 y 2013. Sistema público de Salud. La Pampa, Argentina

\begin{tabular}{|c|c|c|c|c|}
\hline & $\begin{array}{c}\text { MC } \\
(n=29)\end{array}$ & $\begin{array}{c}\text { MNC } \\
(n=58)\end{array}$ & $\begin{array}{l}\text { Comparación } \\
\text { entre grupos }\end{array}$ & Riesgo relativo \\
\hline Mujeres que realizaron consultas de control & $\begin{array}{c}14 \\
(49 \%)\end{array}$ & $\begin{array}{c}25 \\
(43 \%)\end{array}$ & $p=0,6474$ & $\begin{array}{c}1.12 \\
(0,70-1,82)\end{array}$ \\
\hline Mujeres que consultaron por urgencia odontológica & $\begin{array}{c}19 \\
(66 \%)\end{array}$ & $\begin{array}{c}14 \\
(24 \%)\end{array}$ & $p=0,0002$ & $\begin{array}{c}2,71 \\
(1,6-4,5)\end{array}$ \\
\hline
\end{tabular}

La figura 1 muestra la distribución porcentual de consultas de urgencia odontológica según el número de consultas (0 a 4) y si eran MC o MNC. No requirió ninguna consulta de ur- gencia el $34 \%$ de MC, y el 76\% de MNC durante 4 ańos. En el grupo de $\mathrm{MC}$, se realizaron hasta 3 o 4 consultas, situación inexistente en MNC. 
Figura 1: Distribución porcentual de consultas de urgencia en odontología, según MC (n=29)y MNC $(n=58)$

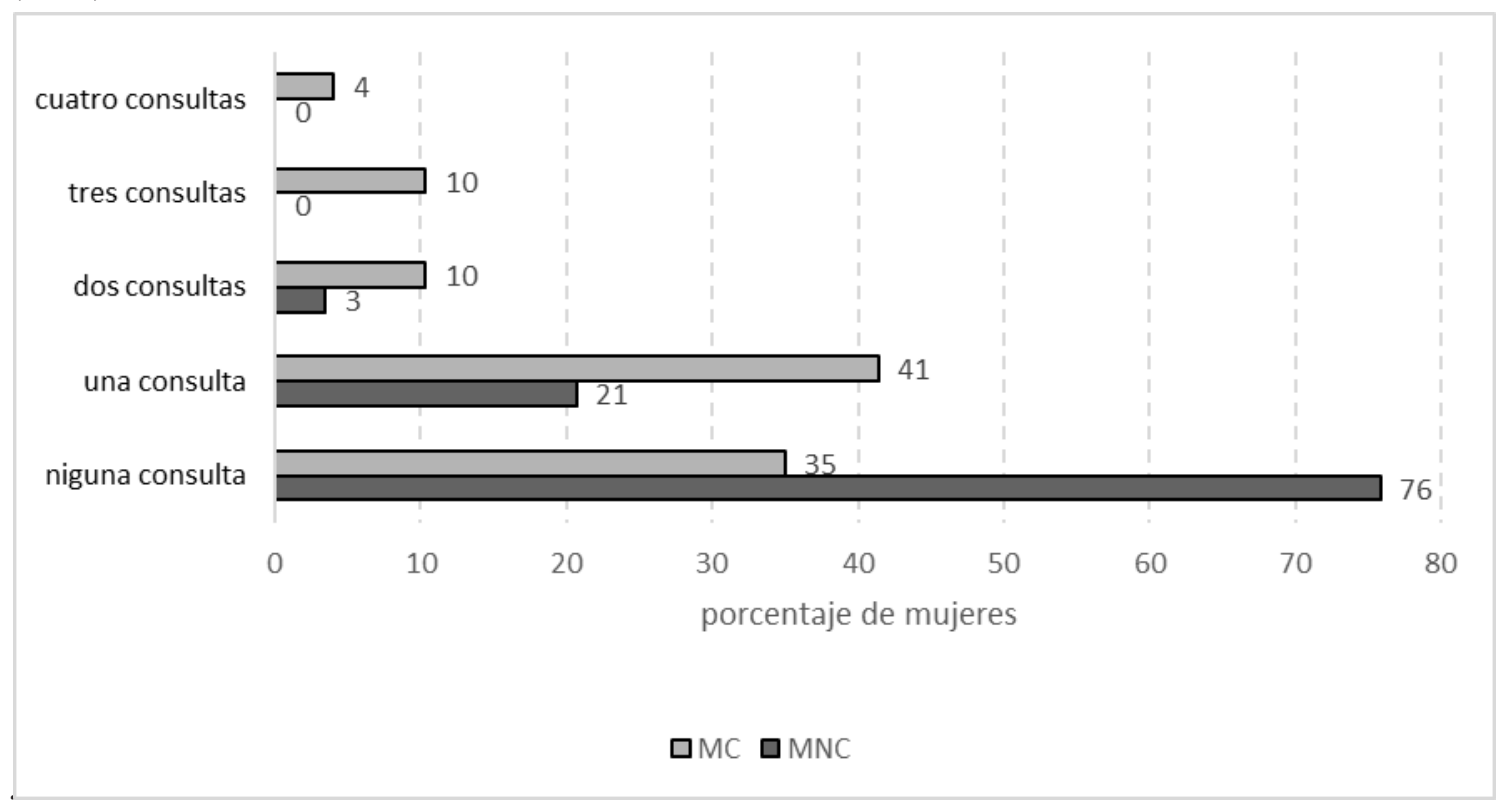

Pueden verse en Tabla 4 los diagnósticos odon- En el grupo de MC, 10/16 (62\%) con diagnóstológicos comparados entre grupos. Las MC tico de gingivitis y periodontitis, tenían menos presentaron una frecuencia significativamen- de 7 años de consumo. Las restantes, tenían ente mayor de gingivitis y periodontitis que las tre 7 y 13 ańos de consumo de cannabis y/o MNC ( $\mathrm{p}=0,0001)$. cocaína.

Tabla 4: Diagnóstico odontológico en consultas de urgencia durante 4 ańos. Comparación entre MC $(n=29)$ y MNC ( $n=58)$. Sistema público de salud de La Pampa, Argentina

\begin{tabular}{|c|c|c|c|c|}
\hline Diagnóstico odontológico & $\begin{array}{c}M C \\
(n=29)\end{array}$ & $\begin{array}{c}\text { MNC } \\
(n=58)\end{array}$ & $\begin{array}{c}\text { Comparación entre } \\
\text { grupos }\end{array}$ & $\begin{array}{l}\text { Riesgo } \\
\text { Relativo }\end{array}$ \\
\hline $\begin{array}{l}\text { Gingivitis y periodontitis } \\
\text { K05 }\end{array}$ & 16 & 6 & $p=0,0001$ & $\begin{array}{c}5,33 \\
(I C=2,20-11,49)\end{array}$ \\
\hline Caries (K02) & 5 & 6 & $p=0,36$ & $\begin{array}{c}1,61 \\
(I C=0,56-5,04)\end{array}$ \\
\hline $\begin{array}{l}\text { Enfermedades de la pulpa y tejidos periapicales } \\
\text { (KO4) }\end{array}$ & 4 & 5 & $p=0,45$ & $\begin{array}{c}1,60 \\
(I C=0,47-5,59)\end{array}$ \\
\hline Pérdida parcial de dientes (K08-4) & 3 & 0 & -- & -- \\
\hline Estomatitis y lesiones afines (K12) & 1 & 0 & -- & -- \\
\hline Celulitis- absceso de boca (K12-2) & 1 & 0 & -- & -- \\
\hline $\begin{array}{l}\text { Otros trastornos de la encía y de la zona edéntula } \\
\qquad \text { (KO6) }\end{array}$ & 0 & 2 & -- & -- \\
\hline
\end{tabular}


Una MC consultó en el servicio de emergencias médicas, y fue internada por celulitis de la cara a punto de partida de infección dentaria. No hubo MNC internadas por patología bucal.

\section{Discusión}

Las mujeres de ambos grupos presentados eran usuarias del sistema público de salud, residían cerca del hospital donde tuvieron el parto, carecían de obra social o prepaga, y tenían similar nivel educativo. Esto es importante, ya que distintos estudios en la población general y en consumidores de DP hallaron peor estado bucal y mayor gravedad de enfermedad periodontal a menor nivel socioeconómico y educativo de los individuos evaluados. ${ }^{(16-19)}$

Como se mostró en la Tabla 3, no hubo diferencias significativas entre grupos en el porcentaje de consultas odontológicas de control. Sí se encontraron diferencias en las consultas de urgencias, que fueron mayores en MC. Además, en $\mathrm{MC}$ el porcentaje de consultas de urgencias superó a las de control (lo contrario que en MNC). Algunos autores han destacado la poca adhesión a realizar controles de salud en consumidoras de cocaína y cannabis. ${ }^{(7,20,21)}$ Ésto también se ha descripto en estudios sobre consultas dentarias en consumidores de DP, con una tendencia a consultar casi exclusivamente ante emergencias. ${ }^{(2,22)}$

El principal diagnóstico de urgencia de las MC fue "gingivitis y periodontitis", significativamente mayor que en MNC. La enfermedad periodontal es la principal consecuencia odontológica del consumo de cannabis y cocaína, aunque en general es referida con tiempos de consumo superiores a 7 o 13 años, mayor a lo descripto en este estudio. ${ }^{(5,8-12,23)}$ Sin embargo, las $\mathrm{MC}$ en su gran mayoría también fumaban tabaco, y la mitad habían consumido alcohol. El policonsumo caracteriza a la mayoría de los estudios sobre cannabis y cocaína, siendo cannabis y tabaco la asociación más frecuente. ${ }^{(10,24)}$ El policonsumo, sumado a otros graves factores sociales y familiares coexistentes, dificultan conocer cuál es el peso de cada uno de ellos, o del conjunto, sobre los resultados presentados. ${ }^{(7)}$ Una limitación del estudio es su carácter retrospectivo, que impide conocer el tiempo de tabaquismo y la intensidad del consumo de alcohol. Otra limitación es que se refiere exclusivamente a mujeres incorporadas al estudio en período posparto. De manera que su salud bucal pudo haber estado influenciada por los cambios hormonales del embarazo, que muchos autores asocian con peor salud periodontal. ${ }^{(25,26)}$

Sin embargo, considerando que hay escasa información sobre la salud bucal de las mujeres consumidoras de cannabis y/o cocaína, este estudio podría ser de utilidad para la salud pública y los profesionales, especialmente considerando que dicho consumo está en aumento globalmente. Podría preverse que se requerirá mayor asistencia odontológica en $\mathrm{MC}$ en próximos años. De hecho, ya en 2012, un estudio detectó que un $21 \%$ de los entrevistados en sala de espera odontológica refirieron consumos problemáticos de alguna DP, en 16,8\% de los casos de cannabis, y en $1 \%$ de cocaína, superando a los porcentajes en la población general. ${ }^{(27)}$

Otra consideración de importancia para la práctica profesional, es que se ha descripto un potencial de riesgo de interacción grave entre algunos anestésicos locales con cannabis. Por estos motivos, sería importante incluir preguntas sobre consumo en el historial de los pacientes. ${ }^{(28)}$

Finalmente, se ha descripto que en el mundo y en Latinoamérica en particular, la cantidad de individuos afectados por periodontitis ha aumentado sustancialmente. ${ }^{(29)} \mathrm{Y}$ recientemente se ha relacionado a la periodontitis con complicaciones como muerte, ingreso a UTI y requerimiento de ventilación mecánica en pacientes infectados por COVID-19. ${ }^{(30)}$ De manera que los gestores de salud, además de los profesionales e investigadores, tendrán que estar atentos a próximas evidencias sobre la relación entre esos 
datos en aumento y los efectos de cannabis, cocaína y del policonsumo.

\section{Conclusiones}

Las MC de cannabis y/o cocaína realizaron más consultas por urgencias, y presentaron con mayor frecuencia diagnóstico de gingivitis y periodontitis, que las MNC. Dado el alto porcentaje de policonsumo, no pueden atribuirse esas diferencias a una u otra droga. Pero este estudio es un aporte para que la salud pública considere la necesidad de atender anticipadamente la salud bucal de las MC, además de abordar la complejidad general de sus varios factores de riesgo coexistentes. El período inmediato posparto podría ser oportuno para hacer un control odontológico y dar pautas preventivas, considerando que posteriormente la tendencia será a consultar mayormente por urgencias odontológicas.

\section{Referencias}

1. Degenhardt L, Hall W. Extent of illicit drug use and dependence, and their contribution to the global burden of disease. Lancet. 2012;379(9810):55-70.

2. Teoh L, Moses G, McCullough MJ. Oral manifestations of illicit drug use. Aust Dent J. 2019;64(3):213222.

3. Secretaria de políticas Integrales sobre Drogas de la Nación Argentina (SEDRONAR). Estudio nacional en población de 12 a 65 ańos sobre consumo de sustancias psicoactivas. Argentina, 2017. [Fecha de acceso: 12 de abril de 2021]. Disponible en: https://www.argentina.gob.ar/sedronar/investigaciony-estadisticas

4. Gigena Pablo C, Bella Marcela I, Cornejo Lila S. Salud bucal y hábitos de consumo de sustancias psicoactivas en adolescentes y jóvenes drogodependientes en recuperación. Odontoestomatología [Internet]. 2012; 14( 20 ): 49-59. [Fecha de acceso: 17 de abril de 2021]. Disponible en: http://www.scielo. edu.uy/scielo.php?script=sci_arttext\&pid=S1688-93392012000200006\&lng=es.

5. Antoniazzi RP, Zanatta FB, Rösing CK, Feldens CA. Association Among Periodontitis and the Use of Crack Cocaine and Other Illicit Drugs. J Periodontol. 2016;87(12):1396-1405.

6. Cury PR, Oliveira MG, Dos Santos JN. Periodontal status in crack and cocaine addicted men: a crosssectional study. Environ Sci Pollut Res Int. 2017 Feb;24(4):3423-3429.

7. Villarreal, M, Belmonte, V, Olivares, JL, \& Abdala, A (2020). Trayectorias sanitarias de mujeres consumidoras de cocaína y/o cannabis durante el embarazo. Estudio de cohorte retrospectivo en La Pampa, Argentina. Revista De La Facultad De Ciencias Médicas De Córdoba; 77(2), 79-85. [Fecha de acceso: 7 de abril de 2021]. Disponible en: https://revistas.unc.edu.ar/index.php/med/article/view/26838

8. Thomson WM, Poulton R, Broadbent JM, et al. Cannabis smoking and periodontal disease among young adults. JAMA. 2008;299(5):525-531.

9. Keboa MT, Enriquez N, Martel M, Nicolau B, Macdonald ME. Oral Health Implications of Cannabis Smoking: A Rapid Evidence Review. J Can Dent Assoc. 2020 Jan;86:k2.

10. Mederos, M., Francia, A., Chisini, L. A., Grazioli, G., \& Andrade, E. Influencia del consumo de cannabis en la enfermedad periodontal. Odontoestomatología 2018; 20(31). [Fecha de acceso: 7 de abril de 2021]. Disponible en http://www.scielo.edu.uy/scielo.php?script=sci_arttext\&pid $=$ S1688-93392018000100004

11. Chisini LA, Cademartori MG, Francia A, Mederos M, Grazioli G, Conde MCM, Correa MB. Is the use of Cannabis associated with periodontitis? A systematic review and meta-analysis. J Periodontal Res. 2019 Aug;54(4):311-317.

12. Chaparro-González NT, Fox-Delgado MA, Pineda- Chaparro RT, Perozo-Ferrer BI, Díaz-Amell AR, Torres V. Oral and maxillofacial manifestations in patients with drug addiction. Odontoestomatología 
2018; 20(32):24-31. [Fecha de acceso: 7 de abril de 2021]. Disponible en: http://www.scielo.edu.uy/ scielo.php?script=sci_arttext\&pid=S1688-93392018000200024\&lng=es

13. Fernández-Martínez, N, Denis-Rodríguez, PB, Capetillo-Hernández, G. (2017) Periodontopatias y lesiones orales en consumidores de cocaína con ingreso reciente a un programa de rehabilitación en relación con pacientes no consumidores. Rev Mex Med Forense 2017, 2(1):19-26 . [Fecha de acceso: 15 de abril de 2021]. Disponible en: https://www.medigraphic.com/pdfs/forense/mmf-2017/mmf171c.pdf

14. Cury PR, Araujo NS, das Graças Alonso Oliveira M, Dos Santos JN. Association between oral mucosal lesions and crack and cocaine addiction in men: a cross-sectional study. Environ Sci Pollut Res Int. 2018 Jul;25(20):19801-19807.

15. Di Rienzo J.A., Casanoves F., Balzarini M.G., Gonzalez L., Tablada M., Robledo C.W. InfoStat versión 2019. Centro de Transferencia InfoStat, FCA, Universidad Nacional de Córdoba, Argentina. [Fecha de acceso: 12 de junio de 2021]. Disponible en: http://www.infostat.com.ar

16. Hakeberg, M., Wide Boman, U. Self-reported oral and general health in relation to socioeconomic position. BMC Public Health 18, 63 (2018). [Fecha de acceso: 21 de abril de 2021]. Disponible en: https://bmcpublichealth.biomedcentral.com/articles/10.1186/s12889-017-4609-9\#citeas

17. Paksoy T, Ustaoğlu G, Peker K. Association of socio-demographic, behavioral, and comorbidity-related factors with severity of periodontitis in Turkish patients. Aging Male. 2020;1:1-10.

18. Borrell LN, Beck JD, Heiss G. Socioeconomic disadvantage and periodontal disease: the Dental Atherosclerosis Risk in Communities Study. Am J Public Health. 2006;96(2):332-339. [Fecha de acceso: 22 de abril de 2021]. Disponible en: https:/www.ncbi.nlm.nih.gov/pmc/articles/PMC1470476/

19. Baskaradoss JK, Geevarghese A. Utilization of dental services among low and middle income pregnant, post-partum and six-month post-partum women. BMC Oral Health. 2020 Apr 20;20(1):120. [Fecha de acceso: 10 de abril de 2021]. Disponible en: https://bmcoralhealth.biomedcentral.com/articles/10.1186/s12903-020-01076-9

20. Magri R, Míguez H, Parodi V, "et al". Consumo de alcohol y otras drogas en embarazadas. Arch. Pediatr. Urug. 2007;78( 2 ):122-132. [Fecha de acceso: 22 de junio de 2021]. Disponible en http://www. scielo.edu.uy/scielo.php?script=sci_arttext\&pid=S1688-12492007000200006

21. Versteeg PA, Slot DE, van der Velden U, van der Weijden GA. Effect of cannabis usage on the oral environment: a review. Int J Dent Hyg. 2008;6 (4): 315-20.

22. Ramo DE, Delucchi KL, Hall SM, Liu H, Prochaska JJ. Marijuana and tobacco co-use in young adults: patterns and thoughts about use. J. Stud. Alcohol Drugs. 2013;74:301-310. [Fecha de acceso: 7 de abril de 2021]. Disponible en https:/www.ncbi.nlm.nih.gov/pmc/articles/PMC3568169/

23. Yazdanian M, Armoon B, Noroozi A, Mohammadi R, Bayat AH, Ahounbar E, Higgs P, Nasab HS, Bayani A, Hemmat M. Dental caries and periodontal disease among people who use drugs: a systematic review and meta-analysis. BMC Oral Health. 2020 Feb 10;20(1):44. [Fecha de acceso: 10 de abril de 2021]. Disponible en: https://pubmed.ncbi.nlm.nih.gov/32041585/

24. Hartnett E, Haber J, Krainovich-Miller B, Bella A, Vasilyeva A, Lange Kessler J. Oral Health in Pregnancy. J Obstet Gynecol Neonatal Nurs. 2016 Jul-Aug;45(4):565-73.

25. Güncü GN, Tözüm TF, Çaglayan F. Effects of endogenous sex hormones on the periodontium - review of literature. Aust Dent J. 2005;50(3):138-145.

26. Brondani MA, Alan R, Donnelly L. Stigma of addiction and mental illness in healthcare: The case of patients' experiences in dental settings. PLoS One. 2017 May 22;12(5):e0177388. . [Fecha de acceso: 18 de abril de 2021]. Disponible en: https://journals.plos.org/plosone/article?id=10.1371/journal. pone. 0177388

27. Ilgen M, Edwards P, Kleinberg F, Bohnert AS, Barry K, Blow FC. The prevalence of substance use among patients at a dental school clinic in Michigan. J Am Dent Assoc. 2012 Aug;143(8):890-6.

28. Schatman ME, Patterson E, Shapiro H. Patient Interviewing Strategies to Recognize Substance Use, Misuse, and Abuse in the Dental Setting. Dent Clin North Am. 2020 Jul;64(3):503-512.

29. Fischer RG, Lira Junior R, Retamal-Valdes B, Figueiredo LC, Malheiros Z, Stewart B, Feres M. Periodontal disease and its impact on general health in Latin America. Section V: Treatment of periodonti- 
tis. Braz Oral Res. 2020 Apr 9;34(supp1 1):e026. [Fecha de acceso: 20 de abril de 2021]. Disponible en https://www.scielo.br/scielo.php?script=sci_arttext\&pid=S1806-3242020000200604

30. Marouf N, Cai W, Said KN, Daas H, Diab H, Chinta VR, Hssain AA, Nicolau B, Sanz M, Tamimi F. Association between periodontitis and severity of COVID-19 infection: A case-control study. J Clin Periodontol. 2021;48:483-491.

\section{Nota declaración de interés:}

No existen conflictos de intereses con otros autores, instituciones, laboratorios, profesionales u otros.

\section{Nota contribución de los autores:}

1. Concepción y diseño del estudio

2. Adquisición de datos

3. Análisis de datos

4. Discusión de los resultados

5. Redacción del manuscrito

6. Aprobación de la versión final del manuscrito

MV, VB, JLO y CL contribuyeron en: 1, 2, 3, 4, 5 y 6.

MPA contribuyó en: 3, 4, 5 y 6 .

\section{Nota de aceptación:}

Este artículo fue aprobado por la editora de la revista Mag. Dra. Vanesa Pereira-Prado. 